(C) [2007] IEEE. Reprinted, with permission, from [Jiaxin Chen, Youguang Guo, Jianguo Zhu and Jianxun Jin, Performance Analysis of a Surface Mounted Permanent Magnet Brushless DC Motor using an Improved Phase Variable Model, Industry Applications Conference, 2007. 42nd IAS Annual Meeting. Conference Record of the 2007 IEEE 23-27 Sept. 2007]. This material is posted here with permission of the IEEE. Such permission of the IEEE does not in any way imply IEEE endorsement of any of the University of Technology, Sydney's products or services. Internal or personal use of this material is permitted. However, permission to reprint/republish this material for advertising or promotional purposes or for creating new collective works for resale or redistribution must be obtained from the IEEE by writing to pubs-permissions@ieee.org. By choosing to view this document, you agree to all provisions of the copyright laws protecting it 


\section{Performance Analysis of a Surface Mounted Permanent Magnet Brushless DC Motor using an Improved Phase Variable Model}

\author{
Jiaxin Chen \\ College of Electromechanical \\ Engineering \\ Donghua University \\ Shanghai, China \\ chjiaxin@dhu.edu.cn
}

\author{
Youguang Guo, Jianguo Zhu \\ Faculty of Engineering \\ University of Technology, Sydney \\ Sydney, NSW, Australia \\ youguang@eng.uts.edu.au \\ joe@eng.uts.edu.au.au
}

\author{
Jianxun Jin \\ School of Automation Engineering \\ University of Electronic Science and \\ Technology of China \\ Chengdu, Sichuan, China \\ jxjin@uestc.edu.cn
}

\begin{abstract}
This paper presents the performance analysis of a high-speed surface mounted permanent magnet (PM) brushless DC motor by using an improved phase variable model. Magnetic field finite element analyses are conducted to accurately calculate key motor parameters such as air gap flux, back electromotive force and inductance, and their dependence on rotor position and magnetic saturation. To evaluate the comprehensive performance of the motor, especially the motor output at high-speed operation, which is affected by the dynamic inductances, an improved phase variable model is derived. In the model, the rotor position dependence of the key parameters is taken into account. The motor prototype has been constructed and tested with both a dynamometer and a high-speed embroidery machine, validating successfully the theoretical calculations.
\end{abstract}

Keywords-permanent magnet (PM) motor; brushless DC (BLDC) motor; surface-mounted; improved phase variable model; numerical magnetic field analysis

\section{INTRODUCTION}

Thanks to their many advantages such as high efficiency, high power density and high drive performance, permanent magnet (PM) brushless DC (BLDC) motors have been widely used in industrial and domestic appliances, and a large amount of work has been conducted for their advanced design and performance analysis [1-2]. However, these analyses are generally based on many simplifications and assumptions such as ideal trapezoidal waveform of back electromotive force (emf), constant winding inductance versus rotor position, negligible cogging torque and core loss. To accurately analyze the BLDC motor, the real waveforms of these parameters should be included [3-4].

For performance evaluation, compared with an equivalent electrical circuit model, the time-stepping nonlinear magnetic field finite element analysis (FEA) can provide accurate results but is more time consuming. A phase variable model of BLDC motor based on FEA and coupled with external circuits, which behaves much faster with the same level of accuracy, has been introduced and verified in [4-5]. In the model, the inductances, back emf and cogging torque were obtained by nonlinear FEA. However, the equation-based model cannot be applied to BLDC directly and an additional model composed of several circuit components has to be employed. To solve this problem, a pure mathematic method is proposed in this paper. By using the method, the central point potential (voltage) of the Y-type three phase windings can be worked out, so that the port voltages of three phase windings can be obtained and the model can be directly applied to BLDC motors. The theoretical procedure is given in detail.

The improved phase variable model has been implemented in the Simulink environment and used to analyze the performance of a high-speed surface mounted PM BLDC motor for driving embroidery machines. In the model, key motor parameters such as winding flux, back emf, inductance and cogging torque are accurately determined based on magnetic field FEAs, which can take into account the details of motor structure and dimensions and the nonlinear properties of ferromagnetic cores. The simulations agree with the experimental results on the motor prototype operated with a BLDC control scheme.

\section{IMPROVED PHASE VARIABLE MODEL OF BLDC MOTOR}

The d-q frame, which does not exist actually, shows no advantage over the abc frame [4]. For a BLDC motor with three symmetrical phase windings of Y-connection without the central line, the equation-based model in the abc frame is given by

$$
\begin{aligned}
& V_{a b c}=r_{a b c} i_{a b c}+\frac{d \psi_{a b c}}{d t}+e_{a b c} \\
& \psi_{a b c}=L_{a b c} i_{a b c} \\
& T_{m}=\frac{e_{a} i_{a}+e_{b} i_{b}+e_{c} i_{c}}{\omega_{r}}+T_{c o g} \\
& J \frac{d \omega_{r}}{d t}=T_{m}-B \omega_{r}-T_{L}
\end{aligned}
$$




$$
\begin{aligned}
& {\left[\begin{array}{c}
\frac{d \psi_{s a}}{d t} \\
\frac{d \psi_{s b}}{d t} \\
\frac{d \psi_{s c}}{d t}
\end{array}\right]=\left[\begin{array}{lll}
\frac{\partial \psi_{s a}}{\partial i_{a}} & \frac{\partial \psi_{s a}}{\partial i_{b}} & \frac{\partial \psi_{s a}}{\partial i_{c}} \\
\frac{\partial \psi_{s b}}{\partial i_{b}} & \frac{\partial \psi_{s b}}{\partial i_{b}} & \frac{\partial \psi_{s b}}{\partial i_{a}} \\
\frac{\partial \psi_{s c}}{\partial i_{a}} & \frac{\partial \psi_{s c}}{\partial i_{b}} & \frac{\partial \psi_{s c}}{\partial i_{c}}
\end{array}\right]\left[\begin{array}{c}
\frac{d i_{a}}{d t} \\
\frac{d i_{b}}{d t} \\
\frac{d i_{c}}{d t}
\end{array}\right]+\left[\begin{array}{c}
\frac{\partial \psi_{s a}}{\partial \theta} \\
\frac{\partial \psi_{s b}}{\partial \theta} \\
\frac{\partial \psi_{s c}}{\partial \theta}
\end{array}\right] \frac{d \theta}{d t}} \\
& =\left[\begin{array}{lll}
L_{a a} & L_{a b} & L_{a c} \\
L_{b a} & L_{b b} & L_{b c} \\
L_{c a} & L_{c b} & L_{c c}
\end{array}\right]\left[\begin{array}{l}
\frac{d i_{a}}{d t} \\
\frac{d i_{b}}{d t} \\
\frac{d i_{c}}{d t}
\end{array}\right]+\left[\begin{array}{ccc}
\frac{d L_{a a}}{d \theta} & \frac{d L_{a b}}{d \theta} & \frac{d L_{a c}}{d \theta} \\
\frac{d L_{b a}}{d \theta} & \frac{d L_{b b}}{d \theta} & \frac{d L_{b c}}{d \theta} \\
\frac{d L_{c a}}{d \theta} & \frac{d L_{c b}}{d \theta} & \frac{d L_{c c}}{d \theta}
\end{array}\right]\left[\begin{array}{l}
i_{a} \\
i_{b} \\
i_{c}
\end{array}\right] p \omega_{r}
\end{aligned}
$$$$
\left[\begin{array}{l}
v_{a} \\
v_{b} \\
v_{c}
\end{array}\right]=\left[\begin{array}{ccc}
r_{a} & 0 & 0 \\
0 & r_{b} & 0 \\
0 & 0 & r_{c}
\end{array}\right]\left[\begin{array}{l}
i_{a} \\
i_{b} \\
i_{c}
\end{array}\right]+\left[\begin{array}{ccc}
L_{a a} & L_{a b} & L_{a c} \\
L_{b a} & L_{b b} & L_{b c} \\
L_{c a} & L_{c b} & L_{c c}
\end{array}\right]\left[\begin{array}{c}
\frac{d i_{a}}{d t} \\
\frac{d i_{b}}{d t} \\
\frac{d i_{c}}{d t}
\end{array}\right]
$$$$
+\left[\begin{array}{ccc}
\frac{d L_{a a}}{d \theta} & \frac{d L_{a b}}{d \theta} & \frac{d L_{a c}}{d \theta} \\
\frac{d L_{b a}}{d \theta} & \frac{d L_{b b}}{d \theta} & \frac{d L_{b c}}{d \theta} \\
\frac{d L_{c a}}{d \theta} & \frac{d L_{c b}}{d \theta} & \frac{d L_{c c}}{d \theta}
\end{array}\right]\left[\begin{array}{l}
i_{a} \\
i_{b} \\
i_{c}
\end{array}\right] p \omega_{r}+\left[\begin{array}{c}
e_{a} \\
e_{b} \\
e_{c}
\end{array}\right]
$$$$
L_{a b}=L_{b a}, L_{b c}=L_{c b}, L_{c a}=L_{a c}
$$

$r_{a}=r_{b}=r_{c}$

$$
i_{a}+i_{b}+i_{c}=0
$$

where $L_{a b c}$ is the inductance matrix and the difference between "apparent" and "differential" definitions is ignored here, $\psi_{s j}$ $(j=a, b, c)$ is the flux linkage of phase winding $j$, and $p$ is the number of pole-pairs. The rest of parameters are used as their conventional meanings. The profiles of $L_{a b c}, e_{a b c}$ and $T_{c o g}$ are obtained from the nonlinear transient FEA solutions, in which the rotor position dependence and the saturation effect are considered.

Fig. 1 illustrated the schematic diagram of a typical drive circuit of BLDC motor, where the electrical potentials (voltages) of terminals $a, b, c$ and $N$ (the central point) are $U_{a}$, $U_{b}, U_{c}$ and $U_{N}$, respectively [6].

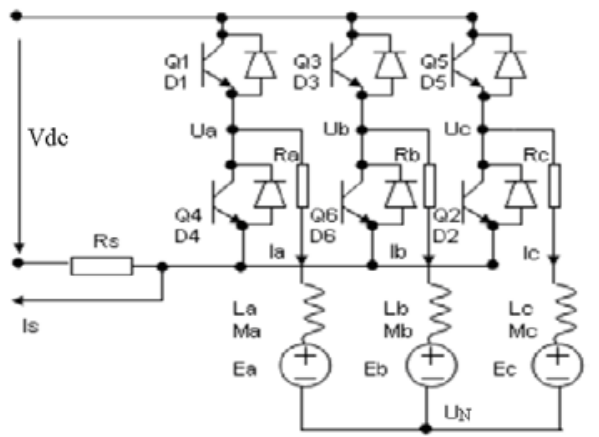

Figure 1. A typical drive circuit for brushless DC motor.

From the above figure, one can obtain

$\left[\begin{array}{l}v_{a} \\ v_{b} \\ v_{c}\end{array}\right]=\left[\begin{array}{l}U_{a}-U_{N} \\ U_{b}-U_{N} \\ U_{c}-U_{N}\end{array}\right]$

Then

$U_{N}=\frac{\sum_{j=a}^{c}\left(U_{j}-v_{j}\right)}{3}$

Substituting (6) into (11) and considering (9), the central point voltage is expressed as

$U_{N}=\frac{1}{3} \sum_{j=a}^{c}\left[U_{j}-\sum_{k=a}^{c}\left(L_{j k} \frac{d i_{j}}{d t}+\frac{d L_{j k}}{d \theta} i_{j} p \omega_{r}\right)-e_{j}(\theta)\right]$

$U_{a}, U_{b}$ and $U_{c}$ are determined by the switching state of inverter with three phases, the state of PWM and the phase currents. When one phase current, e.g. $i_{a}$ of phase $a$, is zero, and the associated circuit is open-circuited (i.e. the winding of phase $a$ is in a non-energized condition), under the consideration of (7)(9), $U_{N}$ and $U_{a}$ can be obtained by

$$
\begin{aligned}
U_{N}= & \frac{\left[U_{b}-L_{b b} \frac{d i_{b}}{d t}-\frac{d L_{b b}}{d \theta} i_{b} p \omega_{r}\right]}{2} \\
+ & \frac{\left[U_{c}-L_{c c} \frac{d i_{c}}{d t}-\frac{d L_{c c}}{d \theta} i_{c} p \omega_{r}\right]}{2}-\frac{\left[e_{b}(\theta)+e_{c}(\theta)\right]}{2}
\end{aligned}
$$

$$
\begin{aligned}
U_{a} & =U_{N}+\left(L_{a a}+L_{b a}+L_{c a}\right) \frac{d i_{a}}{d t}+e_{a}(\theta) \\
& +\left(\frac{d L_{a b}}{d \theta} i_{b}+\frac{d L_{a c}}{d \theta} i_{c}\right) p \omega_{r}+\left(L_{a b} \frac{d i_{b}}{d t}+L_{a c} \frac{d i_{c}}{d t}\right)
\end{aligned}
$$


When the winding current is not equal to zero and PWM is under the state of duty-off, the voltage of input port of phase $a$ can be decided by

$$
\begin{aligned}
& \text { if } i_{a}>0 \text {, then } U_{a}=U_{b u s} \\
& \text { if } i_{a}<0 \text {, then } U_{a}=0
\end{aligned}
$$

where $U_{\text {bus }}$ is the voltage of input power line. According to (13)-(16), one can work out the input port voltages of three phases and their central point, and hence the three phase voltages $v_{a}, v_{b}$ and $v_{c}$.

Referring to (6), the voltage equation of phase $a$ is

$$
\begin{aligned}
v_{a} & =\left(r_{a} i_{a}+L_{a a} \frac{d i_{a}}{d t}\right)+\left(L_{a b} \frac{d i_{b}}{d t}+L_{a c} \frac{d i_{c}}{d t}\right) \\
& +\left(\frac{d L_{a a}}{d \theta} i_{a}+\frac{d L_{a b}}{d \theta} i_{b}+\frac{d L_{a c}}{d \theta} i_{c}\right) p \omega_{r}+e_{a}
\end{aligned}
$$

By defining that

$$
v_{a m}=\left(L_{a b} \frac{d i_{b}}{d t}+L_{a c} \frac{d i_{c}}{d t}\right)
$$

$$
+\left(\frac{d L_{a a}}{d \theta} i_{a}+\frac{d L_{a b}}{d \theta} i_{b}+\frac{d L_{a c}}{d \theta} i_{c}\right) p \omega_{r}
$$

one has

$$
v_{a}=\left(r_{a} i_{a}+L_{a a} \frac{d i_{a}}{d t}\right)+v_{a m}+e_{a}
$$

$$
v_{a}^{\prime}=v_{a}-v_{a m}=\left(r_{a} i_{a}+L_{a a} \frac{d i_{a}}{d t}\right)+e_{a}
$$

Similarly, $v_{b}^{\prime}$ and $v_{c}^{\prime}$ are defined.

According to (1)-(16), a complete Matlab/Simulink-based phase variable model is built as shown in Fig. 2, where $v_{a m}, v_{b m}$ and $v_{c m}, v_{a}, v_{b}$ and $v_{c}, v_{a}^{\prime}, v_{b}^{\prime}$ and $v_{c}^{\prime}$ can be obtained from Matlab functions based on (17)-(20). The rest of work is similar to the modeling of a conventional DC motor, so the proposed model can be easily realized in the Simulink environment.

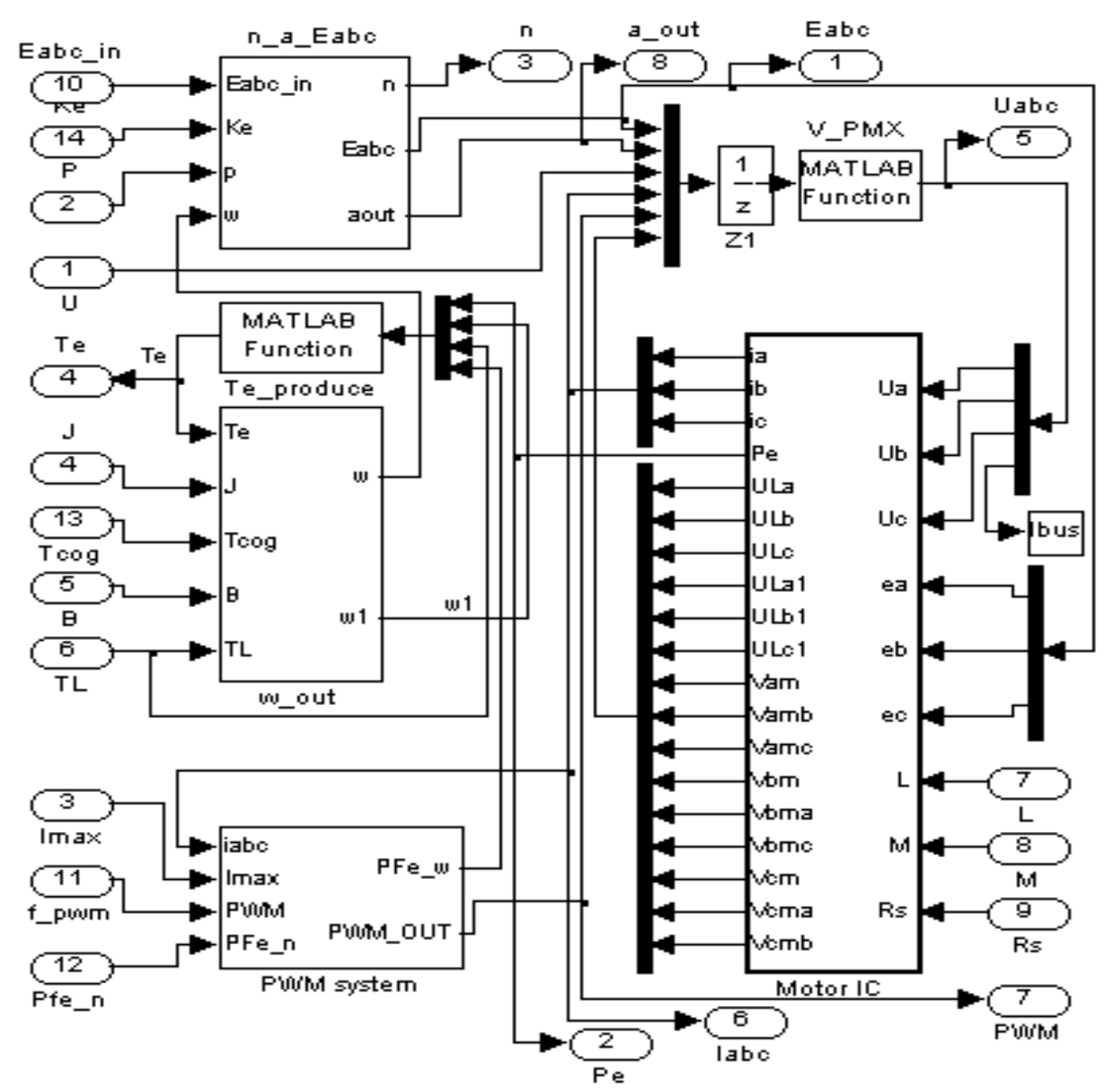

Figure 2. Simulink-based improved phase variable model of BLDC motors. 


\section{PERFORMANCE SimUlation OF A BLDC MOTOR}

Fig. 3 shows the magnetically relevant parts of the PM BLDC motor prototype [7-8]. The laminated stator has 12 slots, in which the three phase single-layer windings are placed (not shown for clarity). The rotor core and shaft are made of solid mild steel, and four pieces of NdFeB PMs are mounted and bound on the surface of the rotor. The stator core has an inner diameter of $38 \mathrm{~mm}$, outer diameter of $76 \mathrm{~mm}$, and axial length of $38 \mathrm{~mm}$. The main air gap length and the height of PMs along the radial magnetization direction are chosen as 1 $\mathrm{mm}$ and $2.5 \mathrm{~mm}$, respectively. The motor is designed to deliver an output torque of $1.0 \mathrm{Nm}$ at a speed of not less than 5000 $\mathrm{rev} / \mathrm{min}$.

Fig. 4 illustrates the plot of magnetic flux density vectors at no-load at $\theta=0^{\circ}$, i.e. the rotor position shown in Fig. 3. From the no-load field distribution, the PM flux (defined as the flux of one coil produced by the rotor PMs), back emf of one phase winding, and cogging torque can be determined. The curves of these parameters against the rotor angular position or time can be obtained by a series of magnetic field FEAs at different rotor positions. Fig. 5 shows the no-load flux linking a coil (two coils form a phase winding) at different rotor positions.

By applying the discrete Fourier transform, the magnitude of the fundamental of the coil flux was calculated as $\phi_{1}=0.543$ $\mathrm{mWb}$, and the emf constant can then be determined as 0.2457 Vs/rad, by

$$
K_{E}=p N_{s} \frac{\phi_{1}}{\sqrt{2}}
$$

where $p=2$ is the number of pole-pairs and $N_{s}=320$ the number of turns of a phase winding. The torque constant can be obtained by $K_{T}=m K_{E}$, where $m=3$ is the number of phases.

From the no-load magnetic field distribution, the cogging torque curve can also be calculated by the Maxwell stress tensor method, or the virtual work method. It was found that the cogging torque of this surface-mounted PM motor is very small with a maximum value of $0.014 \mathrm{Nm}$.

The behavior of the motor equivalent electrical circuit is dominated by the incremental inductance rather than the apparent inductance. In this paper, the winding incremental inductances are calculated by a modified incremental energy method [9], and the results are shown in Fig. 6.

By using the improved phase variable model, comprehensive performances of the BLDC motor can be simulated, such as the curves of speed, current and torque during the start-up or transients when the load or power supply changes. For example, Fig. 7 illustrates the speed curve during the start-up with the full load of $1.0 \mathrm{Nm}$ and the rated inverter voltage of 310 VDC. It can be seen that the motor speed can smoothly increase to the rated speed of $5000 \mathrm{rpm}$. Fig. 8 illustrates the applied voltage, back emf and current of the three phase windings and Fig. 9 shows the bus current waveform.

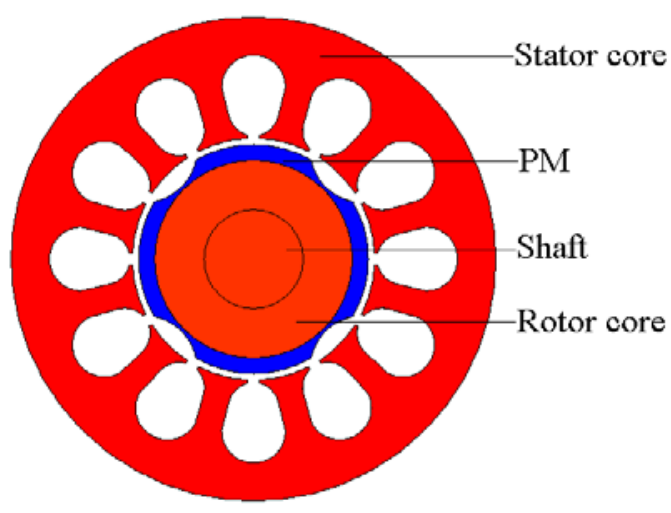

Figure 3. Cross section of a PM BLDC motor.

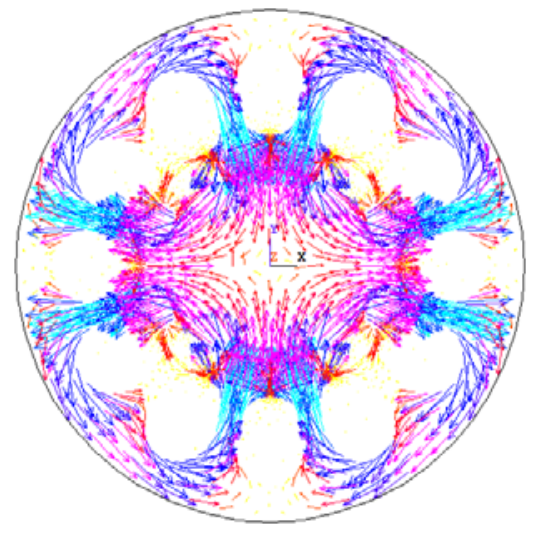

Figure 4. Plot of no-load magnetic flux density vectors.

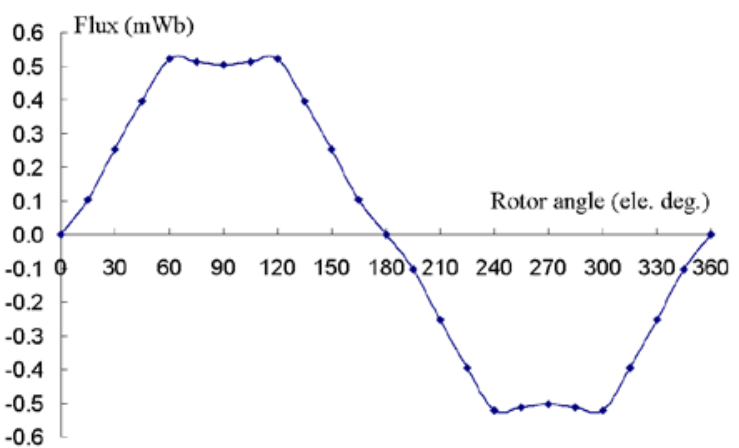

Figure 5. PM Flux per turn versus rotor angle.

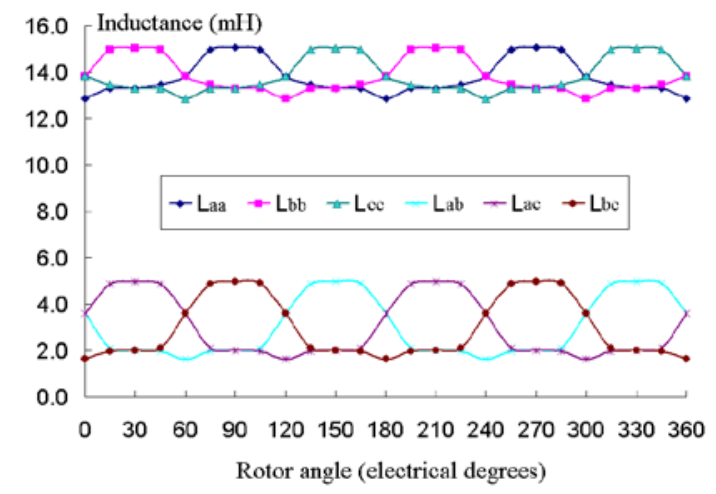

Figure 6. Winding inductances versus rotor angle. 


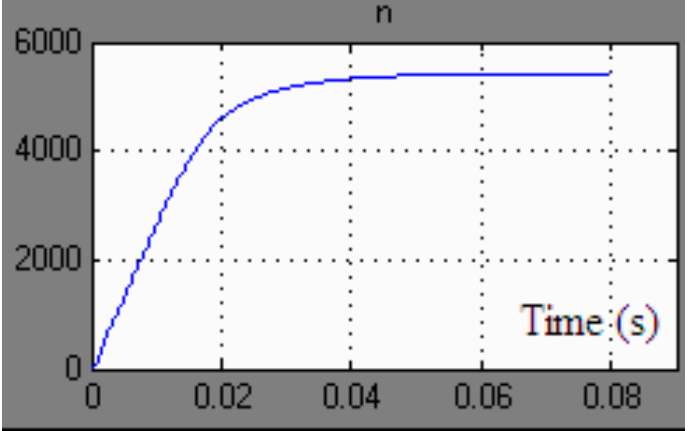

Figure 7. Speed curve during start-up.

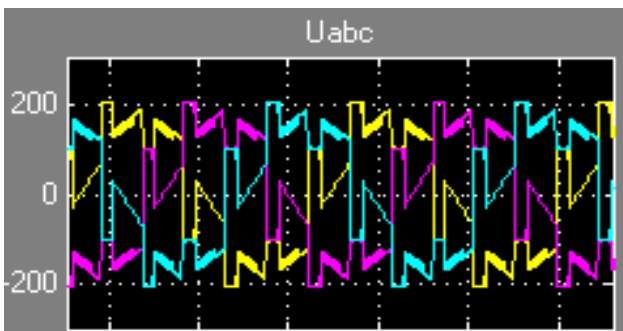

$\begin{array}{lllllll}0.068 & 0.07 & 0.072 & 0.074 & 0.076 & 0.078\end{array}$

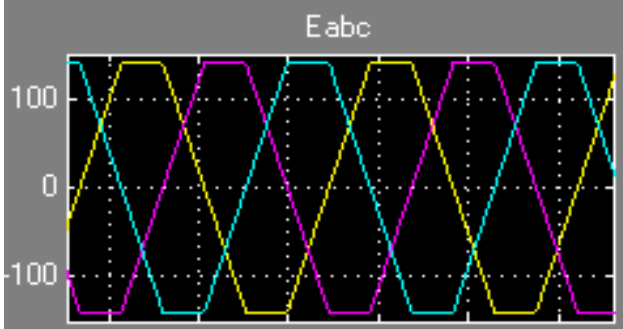

$\begin{array}{lllllll}0.068 & 0.07 & 0.072 & 0.074 & 0.076 & 0.078\end{array}$

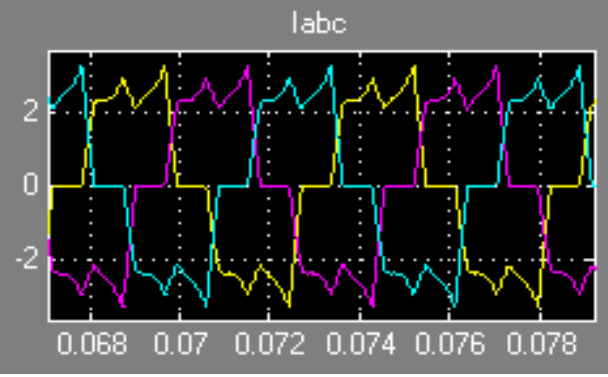

Figure 8. Steady performance (x-axis: Time in s).

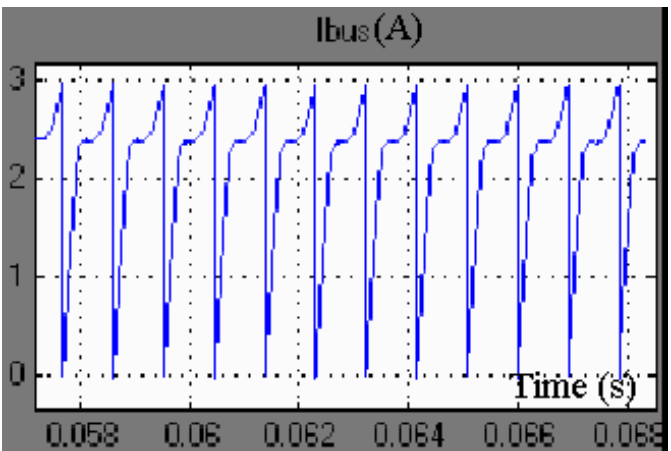

Figure 9. Bus current waveform.

\section{EXPERIMENTAL VALIDATION}

Theoretical computations and simulations are validated by the experiments on the prototype. The back emf, for example, was obtained by measuring the open-circuited terminal voltage when the machine was driven by a DC motor at different rotor speeds and the experimentally determined emf constant is $0.2464 \mathrm{Vs} / \mathrm{rad}$, which is very close to the theoretical value. Fig. 10 shows the measured back emf by an oscilloscope. It can be found that the waveform of back emf is similar to that of the PM flux shown in Fig. 5.

Fig. 11 shows the measured phase current, which agrees with the simulated result in Fig. 8. The steady-state mechanical characteristic is also measured and illustrated in Fig. 12. The measurement was conducted with an input voltage of 220 VAC, corresponding to the inverter voltage of 310 VDC. It can be seen that the motor can operate in a steady speed of no less than $5000 \mathrm{rpm}$ with the rated torque of $1.0 \mathrm{Nm}$.

Other parameters, such as the winding inductances are also in substantially agreement with the theory.

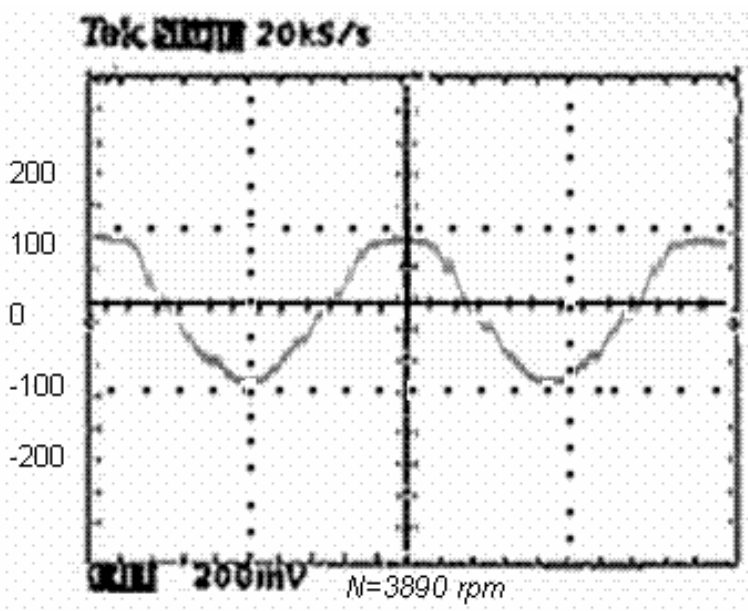

Figure 10. Measured back emf waveform.

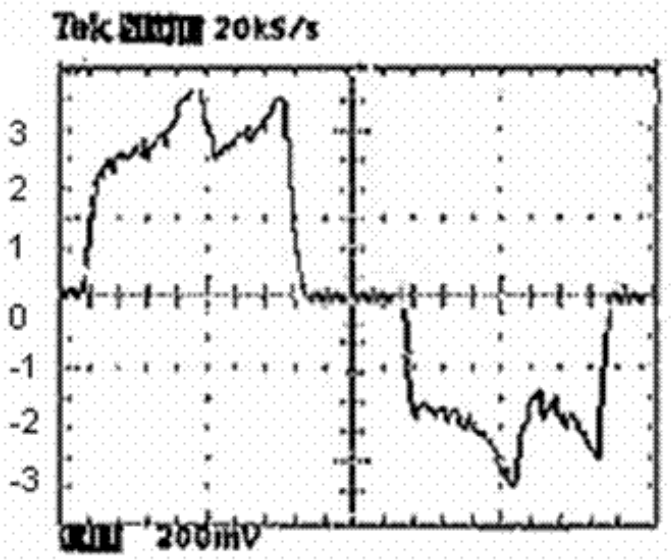

Figure 11. Measured phase current. 


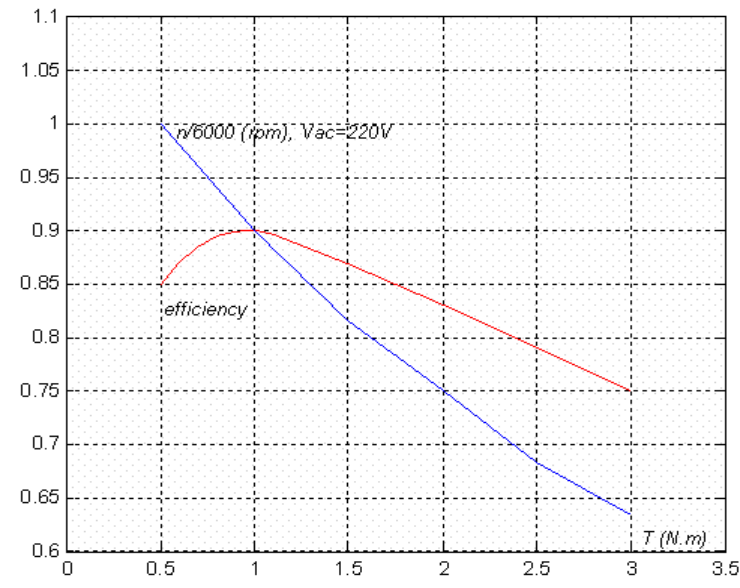

Figure 12. Measured steady-state mechanical characteristic and efficiency.

\section{CONLUSION}

This paper presents an improved phase variable model to evaluate the comprehensive performance of a high speed PM brushless DC motor in both dynamic and steady conditions. A pure mathematical method is proposed to achieve the central point voltage of the Y-connected three phase windings so that the phase voltages can be obtained and the model can be directly applied to analyze the BLDC motor. Key motor parameters are obtained by magnetic field FEAs, in which the rotor position dependence and saturation effect are considered. The data are stored in look-up tables and will be retrieved during the simulation. The presented model has the same accuracy of the full FEA model with much shorter CPU time. The simulations are validated by experiments on the prototype.

\section{REFERENCES}

[1] T. Kenjo, Permanent Magnet and Brushless DC Motors. Oxford: Oxford University Press, 1985.

[2] S. L. Ho, W. N. Fu, H. L. Li, H. C. Wong, and H. Tan, "Performance analysis of brushless DC motors including features of the control loop in the finite element modeling," IEEE Trans. Magn., vol. 37, no. 5, pp. 3370-3374, Sep. 2001.

[3] Y. S. Jeon, H. S. Mok, G. H. Choe, D. K. Kim, and J. S. Ryu, "A new simulation model of BLDC motor with real back EMF waveform," in Proc. the 7th Workshop on Computers in Power Electronics, Blacksburg, VA, USA, July 2000, pp. 217-220.

[4] O. A. Mohammed, S. Liu, and Z. Liu, "A phase variable model of brushless dc motors based on finite element analysis and its coupling with external circuits,” IEEE Trans. Magn., vol. 41, no. 5, pp. 15761579, May 2005.

[5] O. A. Mohammed, S. Liu, and Z. Liu, "Physical modeling of PM synchronous motors for integrated coupling with machine drives," IEEE Trans. Magn., vol. 41, no. 5, pp. 1628-1631, May 2005.

[6] Y. G. Guo, J. G. Zhu, J. X. Chen, and J. X. Jin, "Performance analysis of a permanent magnet claw pole SMC motor with brushless dc control scheme," in Proc. Int. Power Electronics and Motion Control Conf., Shanghai, China, 13-16 Aug. 2006, vol. 2, pp. 1-5.

[7] J. X. Chen, Y. G. Guo, and J. G. Zhu, "Design and performance simulation of a high speed brushless dc motor for embroidery machine application,” in Proc. Int. Technology and Innovation Conf. - Advanced Manufacturing Technology, Hangzhou, China, Nov. 2006. Paper No. 01148.

[8] J. X. Chen, Y. G. Guo, and J. G. Zhu, "An improved phase variable model based on electro-magnetic field coupled with its external circuits for performance evaluation of permanent magnet brushless dc motors," in Proc. Int. Conf. on Industrial Electronics and Applications, Harbin, China, May 2007, Paper ID: P1266.

[9] Y. G. Guo, J. G. Zhu, and H. Y. Lu, "Accurate determination of parameters of a claw pole motor with SMC stator core by finite element magnetic field analysis,” IEE Proceedings - Electric Power Application, vol. 153, no. 4, July 2006, pp. 568-574. 